\title{
Tradition, mémoire et préconstruit chez les militaires américains
}

Anthony Saber

\section{(2) OpenEdition}

1 Journals

Édition électronique

URL : http://journals.openedition.org/asp/642

DOI : 10.4000/asp.642

ISBN : 978-2-8218-0402-9

ISSN : 2108-6354

\section{Éditeur}

Groupe d'étude et de recherche en anglais de spécialité

Édition imprimée

Date de publication : 1 décembre 2006

Pagination : $35-48$

ISSN : 1246-8185

\section{Référence électronique}

Anthony Saber, «Tradition, mémoire et préconstruit chez les militaires américains », ASp [En ligne], 49-50 | 2006, mis en ligne le 11 février 2010, consulté le 22 mars 2021. URL : http://

journals.openedition.org/asp/642 ; DOI : https://doi.org/10.4000/asp.642

Ce document a été généré automatiquement le 22 mars 2021.

Tous droits réservés 


\title{
Tradition, mémoire et préconstruit chez les militaires américains
}

\author{
Anthony Saber
}

\section{Introduction}

1 Sous le double choc du désastre vietnamien et d'une spécialisation technique des personnels sans cesse accentuée, les forces armées américaines ont pu donner le sentiment, dans la décennie 1970, de tourner le dos à l'affirmation de leur identité militaire, pour se transformer en immenses bureaucraties dominées par les ingénieurs et les experts. Cependant, cette convergence avec la société civile, décrite par certains sociologues (Janowitz 1960; Moskos 1970), ne semble être qu'une évolution superficielle, qui n'a jamais remis en cause le fait culturel militaire, dont la prégnance paraitt indéniable. Devenir militaire, c'est traverser une frontière immatérielle, faite de comportements, de rites, de représentations, de symboles. L'entrée dans ce milieu professionnel s'accompagne d'un phénomène de transition, d'acculturation, sans lequel nul ne espérer devenir militaire à part entière. La condition de militaire ne peut pas être décrite comme une simple appartenance administrative : il s'agit avant tout d'une adhésion à une culture spécifique, ou plutôt à un emboîtement de cultures locales, au niveau de l'arme d'abord, puis de la spécialité, et enfin de l'unité; ces périmètres concentriques dessinent les contours de la «militarité»- ce qui différencie radicalement un militaire d'un civil.

2 La prégnance de ce fait culturel est fondée sur le respect de la tradition, mot dont nous constaterons la surprenante polysémie chez les militaires américains. Cette tradition s'appuie à son tour sur des mécanismes de mémoire collective, dont nous tenterons de décrire le fonctionnement. Dès lors, il convient de se demander si le discours militaire n'est pas fortement contraint par cet horizon culturel. Comment, lorsqu'on considère les productions spécifiques aux militaires américains sous l'angle de la langue de spécialité, prendre en compte ce paramètre essentiel de la situation d'énonciation? La 
notion de préconstruit paraît un outil pertinent pour construire une lecture culturelle de ces productions spécifiques.

\section{Le fait culturel militaire}

3 De nombreux indices (récits de formation, ouvrages de FASP) semblent indiquer l'existence d'un fait culturel militaire, soit une culture dont l'acquisition conditionne l'appartenance à ce milieu professionnel spécifique. Nous donnons ici au mot culture, avec Claude Rivière, une acception large, celle d'un mécanisme de production symbolique se fondant sur

[...] l'adhésion à des valeurs, les traditions et coutumes, les modèles de la représentation de soi, les modes de vie et de pensée, mais aussi toutes les créations d'œuvres symboliques. La culture du militaire produit du symbolique autant que du réel. (Rivière in Thiéblemont 1999 : 332-33)

\subsection{Polysémie du mot « traditions »}

Institution souvent décrite comme conservatrice, l'armée américaine s'inscrit dans une tradition dont ses personnels sont le conservatoire vivant. Il faut d'emblée noter la polysémie du mot «traditions » chez les militaires américains. De fait, ce terme décrit d'un même mouvement un lexique particulier, des rites et des coutumes, certaines productions langagières, ainsi que des valeurs exemplaires. Le Naval Historical Center recense ainsi sous cette appellation une liste de termes fréquemment utilisés dans l'US Navy ("Before the mast », " binnacle list », " boot camp »), certaines cérémonies telles que le Navy Birthday, ou le changement de commandant à bord d'une unité, des hymnes officiels de la Marine tels que Eternal Father, Strong to Save ou Anchors Aweigh, mais aussi des comportements rituels tels que Wetting Down a Commission (libations organisées pour fêter des galons nouvellement acquis). On trouve également une description minutieuse de certaines règles d'étiquette (Dining in / Dining out), de même que le système d'attribution des noms aux bâtiments de la flotte. La tradition est donc perçue comme un ensemble disparate de rites, de valeurs, d'objets, de comportements, de réflexes langagiers, soit un faisceau de faits culturels s'inscrivant dans un héritage commun.

5 En somme, l'armée ne se conçoit pas avant tout comme une organisation de professionnels de la violence légitime au sens weberien, mais comme une communauté de culture. Nés fortuitement de l'imagination des militaires ou de rites perpétués par certaines unités, ces faits culturels sont récupérés par l'institution qui, par une démarche patrimoniale et syncrétique, tente d'en faire de puissants instruments d'unité et d'harmonisation culturelle.

6 Le respect de la tradition ne relève pas d'un folklore qui pourrait prêter à sourire, mais d'une pratique quotidienne, rythmant la vie d'une unité. Embarqué sur le sous-marin stratégique USS Nebraska, lors d'une longue patrouille océanique, un journaliste (Waller 2001) se fait l'ethnographe de la vie de l'équipage et constate notamment la scrupuleuse fidélité du commandant aux comportements hérités de la tradition: les dauphins d'or, insigne de spécialité des sous-mariniers, ne sont remis au rookie qu'après avoir chanté l'hymne honorant l'Université du Nebraska. L'étiquette au carré du commandant est strictement observée, les officiers arrivant en retard pour raison de 
service demandent au commandant la permission de s'asseoir. Il est interdit de parler de religion ou de politique.

7 La fonction cohésive de la tradition permet donc d'éviter l'éclatement d'une unité en chapelles rivales composées de techniciens; de fait, l'inévitable spécialisation des métiers militaires menace de faire pièce aux facteurs d'unité, comme le déplore cet officier de l'USAF :

Military customs, courtesies, and traditions are vitally important to our Air Force because shared traditions are one of the few things remaining that can bind officers of widely differing skills and specialties into one professional corps. (Hall 1987)

La tradition crée un lien social, permet d'éviter l'atomisation du corps militaire en sous-chapelles de « techniciens en uniforme ».

\subsection{Typologie des rites militaires}

Cette tradition s'appuie sur un ensemble de rites qui ponctuent la vie militaire. À travers eux, les personnels réaffirment leur adhésion au système de valeurs constitutif de leur identité. Au premier rang de ces rites, on trouve les cérémonies, dont la finalité cohésive est évidente :

La cérémonie collective a les effets d'une tradition en ce qu'elle répète, dans un style rigide, des formes et contenus de représentations que les participants ont en commun. (Rivière 1988 : 141-142)

G. Balandier (1992: 19) souligne également l'utilité des cérémonies comme champ de projection de valeurs : à travers elles, un certain milieu professionnel se montre idéalement sur le mode spectaculaire. Cependant, par rite il faut entendre non seulement une fête ou une cérémonie, mais aussi tout acte solennel à forte charge symbolique. Très répandus au sein des unités, les rites de passage permettent ainsi de marquer l'accession à la condition de militaire, ou à un nouveau statut au sein du corps militaire : la remise d'un décret de promotion à un grade supérieur, d'un diplôme sanctionnant une nouvelle qualification, ou d'un insigne à épingler sur l'uniforme doit s'accompagner de « ritèmes » réaffirmant l'unité et la cohésion du milieu qui accueille l'impétrant:

In the old days, when a seaman was awarded his dolphins, its metal spikes were pounded into his chest - a barbaric ritual Marine Corps paratroopers also practiced. It is now outlawed. (Waller $2001: 15$ )

11 Cérémonies et rites de passage s'intègrent dans un ensemble complexe de matériaux symboliques. Si nous prenons l'exemple du Marine Corps (USMC), dont la «militarité » et la cohésion sont souvent réaffirmées par opposition aux autres armes (considérées plus proches du monde civil), on constate l'existence de 4 types de rites :

- Rites langagiers : par exemple, des locutions telles que «I wish... », « I desire... », lorsqu'elles sont prononcées par un supérieur hiérarchique, doivent être interprétées comme des ordres formels ;

- Rites cérémoniels : les Marines occupent toujours la droite d'une formation ou la tête d'une colonne lorsqu'ils défilent conjointement avec des éléments de l'US Navy ou de l'US Coast Guard;

- Rites comportementaux : chaque année, le 10 novembre, les unités de l'USMC célèbrent la fondation du Corps en 1775 ; la cérémonie comprend toujours la lecture d'un passage du Manuel du Corps (ainsi élevé au rang de texte révélé) ainsi que d'un message du Commandant (le chef d'État-major de l'USMC). Chaque commandant d'unité est chargé de 
découper un gâteau d'anniversaire, dont les deux premières parts sont invariablement offertes au Marine le plus jeune et au Marine le plus âgé de l'assistance, symbolisant ainsi la pérennité du Corps ;

- Rites iconiques ou d'adoration : de loin les plus nombreux, ils renvoient au passé du Corps à travers la sanctification d'objets symboliques ; ainsi les officiers et officiers mariniers du Corps portent un galon amarante (" the Blood Stripe ») sur leurs pantalons, en souvenir du sang versé par les Marines lors de la prise du Fort Chapultepec à Mexico en septembre 1847.

L'institutionnalisation de certains rites les élève au rang de pratiques cohésives et les placent au cœur d'une culture partagée. La charge symbolique qu'ils projettent permet à l'armée de réaffirmer son unité et de produire un discours exaltant les valeurs exemplaires du combattant. La grammaire des rites proposée par Claude Rivière pour décrire les rites politiques nous paraît pertinente ici : ayant pour finalité de réitérer ou de renforcer des liens groupaux, de renouveler ou de revivifier des croyances, de propager les idées d'une culture, de délimiter des rôles, les rites s'organisent selon une structure de "ritèmes »: phases temporelles, rôles des organisateurs, acteurs et spectateurs, nature des valeurs projetées, nature des moyens (lieux, objets, gestes, attitudes) utilisés, structure des communications (sélection des émetteurs et récepteurs selon une hiérarchie précise).

En appliquant ce dernier critère de classement aux rites militaires, nous constatons que, dans leur grande majorité, ils ont pour destinataires la communauté militaire ellemême, et non la société civile. Les rites du Marine Corps peuvent ainsi être décrits comme autoréférentiels, dans la mesure où ils visent presque exclusivement les personnels du Corps. À leur rôle intégrateur s'ajoute donc une fonction différenciatrice : la militarité trouve dans l'autoréférentialité de ces rites une promesse de continuité, de résistance à une dissolution dans le monde civil, et se voit presque érigée en sacré moderne, soit :

[...] le domaine du préservé, du réservé, de l'autorité impérative, du soi-disant légitime indubitable. (Rivière $1988: 146$ )

Toute étude du discours militaire doit par conséquent tenir compte de ces rites, qui, comme le souligne $\mathrm{C}$. Rivière, constituent presque un langage puisqu'ils mobilisent des signes standardisés pour devenir un système de communication symbolique entre membres d'une même communauté. L'échange de sens au sein des milieux militaires américains ne passe donc pas uniquement par des systèmes d'énoncés, mais aussi par une ritologie strictement codifiée.

\subsection{La militarité, système de valeurs et de représentations}

Fondée sur une grammaire des rites et des coutumes, la culture militaire est le champ de projection de certaines valeurs caractéristiques de la militarité. Tentant de définir les valeurs dominantes au sein du corps des officiers de l'USAF, un colonel dresse un catalogue sans surprise : intégrité, sens du devoir, excellence, patriotisme, bravoure au feu, amour du travail, etc. Cependant, l'auteur apporte une précision intéressante :

Career-field identity plays a strong role in our ethos. It has been well advertised that we tend to think of ourselves as navigators, logisticians, pilots, cops, and so forth, instead of as Air Force officers. (Hall 2001) 
Il existe donc des sous-ensembles culturels clairement différenciés au sein de la famille militaire. Les unités de mêlée, plus directement confrontées à l'utilisation de la violence, sont traditionnellement celles dont la culture cohésive est la plus forte.

Parce qu'elle a quelques chances d'être confrontée à la violence désintégratrice, la vitalité du corps militaire est peut-être plus dépendante de sa culture que celle d'autres corps sociaux. Là où la culture est puissante et où elle déborde la trame de plus en plus serrée des règles édictées par l'institution, le corps est d'élite. (Thiéblemont 1999 : 3)

17 Avérée chez les militaires français et américains, cette corrélation entre intensité du fait culturel et caractère guerrier de l'unité fonde des mécanismes différenciateurs. L'espace social militaire semble traversé de fractures : le militaire aura en permanence à sa disposition plusieurs cultures identitaires. Selon les circonstances, tel officier se percevra par exemple alternativement comme ancien élève d'Annapolis, comme artilleur, comme membre d'équipage du bâtiment sur lequel il est affecté, etc. Toutes ces cultures locales se posent "contre" d'autres cultures concurrentes (artilleurs contre transmetteurs, anciens d'Annapolis contre officiers issus du ROTC, etc.). Ces multiples clivages pourraient donner le sentiment d'un manque d'unité, d'une atomisation extrême. En réalité, l'appartenance au milieu professionnel militaire se fonde sur une double légitimation : légitimation différenciatrice, par la multiplication de mécanismes identitaires rivaux, légitimation cohésive par la préservation et le culte d'une mémoire commune.

\section{Fonction cohésive de la mémoire}

C'est par un renvoi au passé, à la mémoire, que les milieux militaires américains assurent leur cohésion. Ce culte de la mémoire s'appuie d'abord sur un effort de préservation du patrimoine culturel commun. La démarche patrimoniale des forces armées américaines en matière culturelle trouve un prolongement institutionnel: l'archivage de la mémoire commune est confié à des historiens en uniforme, qui se font les annalistes des campagnes militaires. C'est ainsi que, venant à peine de s'emparer d'une base militaire irakienne lors de la guerre du Golfe, un lieutenant commandant un escadron de tanks reçoit une visite surprenante :

Out of the blue, a master sergeant came over to my tank carrying a tape recorder and a notepad. He told me that he was an army historian. I thought he had lost his marbles. There was no way a historian would be here so soon. I made a radio call in order to verify the historian's identity and then we sat down on top of my tank and talked. (Vernon et al.1999: 223)

19 De même, les unités nouvellement créées (et donc dépourvues de mémoire propre) sont dotées de matériels symboliques, historiées (les unités d'hélicoptères de l'US Army, fondées au début des années 1960, prennent par exemple l'appellation de cavalerie). Dans l'US Navy, chaque bâtiment reçoit un nom dont la charge symbolique reflète son importance et son prestige: les porte-avions portent souvent le nom d'un président (USS George Washington, USS Reagan), les HVU (High-Value Units, soit les sous-marins stratégiques, les croiseurs, etc.) le nom d'une grande ville ou d'un État (USS Los Angeles) ou même d'épisodes militaires glorieux (USS Shiloh, USS Valley Forge). (on citera par exemple celui du US Naval Institute à Annapolis), grâce auxquels l'armée 
archive systématiquement les témoignages oraux des militaires retraçant leur carrière, leurs espérances, leurs souvenirs. Le patrimoine ainsi préservé fonde une généalogie permettant de légitimer les comportements actuels par un renvoi au passé :

C'est le propre des mythologies, même modernes, que de mettre en connexion

l'actuel avec l'ancien, l'ordre avec le système des pouvoirs à l'origine de

l'institution. (Rivière in Thiéblemont 1999 : 332-33)

L'exaltation de la mémoire permet d'affirmer des continuités réelles ou recréées: l'enjeu principal du milieu professionnel étant sa survie sur le champ de bataille, synonyme de victoire des armes américaines, le renvoi au passé vaut promesse de continuité, de non-anéantissement.

\subsection{Non écrit, coutume et tradition orale}

Ce constant renvoi à l'ancien ne s'appuie pas toujours sur un système d'énoncés ou un ensemble de rites. Les milieux militaires américains se caractérisent en effet par la grande place accordée au non-écrit, à la coutume, à la tradition orale.

On fait ainsi confiance au signe pour dire le passé de chacun: sur l'uniforme des militaires américains, une signalétique complexe retrace les décorations obtenues, mais aussi les formations suivies ou les campagnes accomplies. De même la présence de codes non écrits structure la vie militaire : très souvent, les décisions sont légitimées par référence à une mémoire maintenue. Ainsi, l'US Navy a toujours fait montre d'une grande allergie à la doctrine, à la codification par écrit de ses principes tactiques. Le passage par l'écrit et le règlement semblait aller à l'encontre de la nécessaire autonomie des commandants de navire. Les concepts d'emploi des forces au sein de la Navy ont donc longtemps fait l'objet d'une tradition orale, et, si la revue Proceedings fait office de forum doctrinal, il faut attendre 1993, soit presque deux siècles après la fondation de la Marine, pour voir la naissance du Naval Doctrine Command à Norfolk, organisme chargé de la codification des doctrines navales (qui se heurtera d'ailleurs à la sourde opposition de nombreux amiraux).

\subsection{Esprit de corps et ethos militaire}

L'omniprésence de la mémoire trouve des prolongements sur la scène énonciative. Pour s'inscrire dans les modèles hérités et légitimer ainsi son appartenance à son arme ou à son unité, le militaire américain cherchera à projeter dans son discours une certaine image de soi - un certain ethos destiné à démontrer sa qualité de militaire aux destinataires de ses énoncés. L'objectif est ici, par un certain positionnement énonciatif fondé sur la projection de valeurs figées par la mémoire commune, de réaffirmer la primauté de l'esprit de corps, comme l'indique ce passage tiré d'une fiction à substrat professionnel :

"We Marines are all in this together," he said [...]. "When one man slips off the rope, we'll grab him on the way down. We'll all hang together and we'll do whatever we have to do to get the job done. The Corps is bigger than all of us, and once you are a part of it, you're part of it forever. [...] If you die, when you die, the Corps goes on. It's sorta like a church." (Coonts $1994: 141)$

Pour reprendre un des termes décrivant (avec le logos et le pathos), dans la triade aristotélicienne, les qualités du rhéteur, l'ethos semble être l'outil de prédilection du militaire américain pour projeter dans ses énoncés les valeurs et caractéristiques 
archétypales de son milieu professionnel. La scène énonciative comportera donc chez les militaires américains un certain nombre de comportements langagiers attendus, que l'on pourrait grouper sous l'appellation «tall-tale », par opposition au diplomatique hedging si souvent décrit chez les scientifiques. Parmi d'autres productions portant la trace de cet ethos, quelques titres d'articles doctrinaux parus dans Proceedings reflètent par leur formulation la volonté de l'auteur de démontrer au co-énonciateur son adhésion aux valeurs de référence du milieu militaire: désinvolture et humour (Allelectric ship: Sirloin or just sizzle? ${ }^{1}$; It's the air plan, stupid $\left.{ }^{2}\right)$, irrévérence envers la hiérarchie ou le pouvoir civil (Weinberger-Powell doctrine doesn't cut it ${ }^{3}$ ), réaffirmation de la discipline et de la hiérarchie sur le mode tautologique (The leaders must lead ${ }^{4}$ ).

La mobilisation de ces valeurs stéréotypées contribue à créer une image discursive de soi qui délimite un dedans (le milieu militaire) et un dehors (le monde civil). La communication entre militaires se déroule sur une scène énonciative, théâtre d'une constante stratégie de légitimation. L'ethos militaire permet à l'énonciateur de justifier son discours en tant que pierre supplémentaire ajoutée à cet édifice collectif qu'est l'esprit de corps.

\subsection{La mémoire institutionnelle des unités}

Toutefois, les milieux militaires ne présentent pas au regard un aspect monolithique. $\mathrm{Si}$ les personnels de chaque arme partagent des matériels symboliques communs qui cimentent leur cohésion, ceci n'exclut en rien la présence d'aires culturelles locales, au niveau de l'unité, de l'escadron, du bâtiment. Le prestige d'une unité est d'ailleurs souvent reflété par la densité de son matériel symbolique, qui renvoie immanquablement à un passé riches en actes héroïques et en batailles victorieuses. C'est ainsi que Colin Powell, nommé à l'État-major de la prestigieuse $101^{\mathrm{e}}$ division aéroportée (Powell 1995), s'enthousiasme de rejoindre une unité de légende ( $a$ storybook outfit »), dont le général Lee, son premier commandant, avait déclaré, 4 jours après sa création en août 1942, qu'elle n'avait pas encore d'histoire mais « un rendezvous avec le destin ", illustrant ainsi le parallèle entre prestige militaire et mémoire. Notons le caractère pérenne de ces cultures locales, qui résistent même à un renouvellement complet des personnels de l'unité. Très affirmée au sein de la Marine nationale française (Dufoulon 1998), cette permanence de cultures propres à chaque unité est également perceptible chez de nombreux militaires américains: l'appartenance à un sous-ensemble donné est soulignée par l'adhésion à des représentations et attitudes communes, mais aussi par des signaux de reconnaissance langagiers :

The [24th Infantry] Division had been given its nickname by liberated citizens after Gen. Douglas Mac Arthur announced the end of the Philippines campaign on 5 July 1945. Thereafter, the Victory Division had marked its vehicles and helmets with the $\mathrm{V}$ symbol. In our generation, a soldier of the division saluting a ranking officer greeted the superior with the division motto, "First to Fight, sir," the latter returned the salute with "victory." (Vernon et al. 1999 : 22)

Ces codes langagiers participent de l'ethos décrit plus haut, non plus pour faire le départ entre monde civil et milieux militaires, mais pour établir au sein de ces derniers des hiérarchies traversant les armes, les spécialités, les unités. Ces discours symboliques, mis en compétition, n'entachent en rien la cohésion de l'ensemble : les revendications identitaires et différenciatrices de chaque sous-groupe s'additionnent 
mais ne s'annulent pas, elles traduisent la vitalité d'un corps social traversé de fractures mais néanmoins uni autour de la mémoire et de la tradition.

\section{Le préconstruit, paramètre de la situation d'énonciation}

produits par les milieux militaires américains. Il nous paraît possible de rapprocher cet horizon culturel omniprésent de la notion de préconstruit, élaborée par P. Henry et développée par M. Pêcheux. Les jeux langagiers propres à chaque unité mettent en scène la trace, dans l'énoncé, d'un discours antérieur; c'est ainsi qu'un sergentinstructeur s'étonne de ne pas voir un visiteur se prétendant ancien parachutiste lui fournir la réponse qu'il attend :

“With your permission, Sir?" Sergeant Tannley barked.

"Carry on, Sergeant," Finton said.

"Yes, Sir!” Tannley barked. “Airborne, Sir!”

Finton knew that there was an expected response to that, but he had no idea what it was. He searched his mind desperately for a moment and came up with nothing.

"Right!" he barked.

Sergeant Tannley gave him a look of surprise and wonderment and finally, Finton thought, of suspicion. (Griffin $1987: 102-103$ )

On observe ici, dans l'attente déçue du sergent (tout parachutiste qui se respecte sait qu'à l'interjection « airborne » doit répondre en écho la locution « all the way! »), un effet de préconstruit, soit :

un décalage, par lequel un élément fait irruption dans l'énoncé comme s'il était pensé « avant, ailleurs, indépendamment » (Pêcheux 1975 : 221)

Plus généralement, il nous semble que la prégnance des schémas culturels décrits plus haut limite l'autonomie de l'énonciateur au sein des milieux militaires américains. La communication en leur sein s'appuie en effet souvent sur des évidences partagées par les co-énonciateurs (représentations, éléments symboliques). À cet égard, Pêcheux percevait surtout le préconstruit comme une dépendance du sens vis-à-vis de l'idéologie :

Le caractère matériel du sens, masqué par son évidence transparente pour le sujet, réside dans sa dépendance constitutive à l'égard de ce que nous avons appelé «le tout complexe des formations idéologiques ». (Pêcheux 1975 : 224)

Il semble possible d'adapter cette formulation aux milieux militaires américains, mais c'est ici la culture militaire qui fera office d'horizon idéologique. Se matérialise alors une dépendance étroite entre l'énonciateur et une "formation discursive », soit, selon Pêcheux, l'autorité déterminant tous les énoncés autorisés et souhaitables au sein d'une formation idéologique.

\subsection{L'articulation entre énonciateur et milieu professionnel}

Cette étroite dépendance entre l'énonciateur et le milieu à partir duquel il parle se manifeste d'abord par une contagion entre le style de vie militaire et certains comportements langagiers. Si l'armée moderne ne peut plus être considérée comme une «institution totale» au sens où l'entendent les sociologues (ces institutions prisons, pensionnats, couvents -ayant pour caractéristique commune la prise en charge 
de l'ensemble des aspects de la vie: travail, alimentation, mais aussi loisirs, hébergement, etc.), il n'en reste pas moins qu'elle impose à ses membres des contraintes sensiblement plus fortes que d'autres milieux professionnels. Dès lors, il ne faut pas s'étonner qu'un style de vie devienne un mode d'individuation qui trouve parfois d'amusantes ramifications langagières :

The 2nd Infantry Brigade was part of STRAC, the Strategic Army Corps, composed of elite units prepared to fight on any front at short notice. We used the acronym interchangeably as a noun and an adjective. STRAC was a state of being, a sharpness, a readiness, an esprit de corps. "Sergeant, is the platoon STRAC?" "Yes, sir, we're STRAC.") And, as often happens in the Army, we overdid it. Style overran substance. Being STRAC came to mean looking sharp more than being combatready. (Powell 1995 : 54)

L'articulation entre l'énonciateur et son milieu professionnel se manifeste également par des énoncés attendus. En premier lieu, certaines séquences d'énoncés sont strictement codifiées par l'institution pour constituer une étiquette verbale (enseignée systématiquement aux jeunes recrues dès le boot camp): ainsi le fameux sandwich sir (Sir! + énoncé + Sir!) que tout militaire effectuant ses "classes » est tenu d'utiliser en s'adressant à un supérieur. Il ne faut toutefois pas négliger la portée symbolique de cette étiquette, perçue comme une composante essentielle de la militarité ; à un colonel prêchant les vertus des valeurs militaires traditionnelles et déconseillant notamment à des jeunes lieutenants en formation d'appeler leurs supérieurs par leur prénom, un jeune officier fait part de sa déception :

Sir, I'm an engineer and I work for a major who keeps telling me to call him 'Jim.'

Sir, If I'd wanted to call my engineering boss 'Jim,' I'd have gone to work for

Hewlett-Packard! (Hall $1987: 49$ )

Mais au-delà de ces énoncés figés et quelque peu mécaniques, le préconstruit se manifeste d'abord au plan sémantique: sur une scène énonciative polarisée par les schémas culturels décrits plus haut, l'implicite devient primordial. Certaines productions exploitent d'ailleurs stratégiquement cet effet de préconstruit et doivent donc faire l'objet d'un décodage ou d'une explicitation. Ainsi, le discours médiatique des armées repose sur une stratégie d'atténuation sémantique dont les exemples sont biens connus : collateral damage pour "civils tués par erreur ", MIA (Missing In Action) pour "morts au combat", etc. Mais c'est surtout dans des productions en situation fermée, visant les membres des milieux militaires en non la presse ou l'opinion publique, que l'effet de préconstruit se manifeste avec le plus de vigueur. Le décodage des énoncés demande ici une bonne connaissance des traces presque imperceptibles d'un discours antérieur. Alors affecté à l'État-major de la $101^{\mathrm{e}}$ Division aéroportée, Colin Powell décèle ainsi dans un rapport d'évaluation particulièrement élogieux à son égard l'arrêt de mort de sa carrière :

The narrative evaluation, however, was nearly fatal. He had praised my performance solely as a "trainer." My command potential was ignored. I had not been sent to Fort Carson to become a trainer, but to qualify as a division commander. I had attended division prep school and, in his judgment, had flunked. (Powell 1995 : 260)

Considéré en tant que genre, le rapport d'évaluation militaire comportera une grande variabilité sémantique selon le positionnement respectif des co-énonciateurs. Certains systèmes d'énoncés propres aux militaires exploitent donc directement l'effet de préconstruit. Faut-il pour autant en déduire avec Pêcheux que l'appartenance de l'énonciateur à une formation discursive limitera son autonomie au point de réduire 
l'énoncé au statut d'excipient se positionnant avant tout par rapport à un "déjà-là " omniprésent? La prégnance de la culture militaire pourrait alors réduire tout acte communicatif à un jeu de dupes :

Le fonctionnement d'une formation discursive s'appuie sur un «espace de reformulation-paraphrase " où se constitue l'illusion nécessaire d'une « intersubjectivité parlante » par laquelle chacun sait par avance ce que l' " autre »

va penser et dire. (Pêcheux 1975 : 237)

Dans cette perspective, l'énonciateur ne ferait que sélectionner des séquences d'énoncés déjà disponibles, autorisés par sa formation discursive. Cela dit, nous ne pensons pas que les contraintes résultant de la culture militaire limitent à ce point l'autonomie énonciative des militaires américains - sans exclure pour autant un fort degré de normage et de contrainte. Nous avons déjà constaté, par exemple, la grande liberté de ton des articles doctrinaux (dont le paratexte comporte d'ailleurs souvent un disclaimer indiquant que les opinions exprimées n'engagent que l'auteur). En réalité, c'est surtout sur le plan lexical et terminologique que ces contraintes se manifestent.

\subsection{Des choix lexicaux et terminologiques contraints}

Comme toute activité technique, le métier des armes exige la maîtrise d'une terminologie liée aux équipements, aux procédures tactiques, aux grades, etc. Le degré de spécialisation de cette terminologie est parfois tel que même un jeune officier de Marine fraîchement émoulu d'Annapolis n'y perçoit qu'ésotérisme et mystère :

Volonino was sent to the USS Bluefish, a nuclear attack sub patrolling the Atlantic. He felt overwhelmed at first. All around him were junior officers only three or four years older than he, who were operating this complex machine and rattling off nautical terms in what sounded like a foreign language. (Waller $2001: 49$ )

Cette terminologie se double cependant de microlectes, ou de terminologies locales, dont l'existence a été également soulignée au sein de l'armée de terre française (Paveau 1994). Propres à un sous-ensemble donné (unité, escadron, bâtiment...), ces microlectes n'ont pas de justification technique: ils fonctionnent comme des rites intégrateurs. Ainsi, le terme «STRAC » cité plus haut montre qu'un simple acronyme peut se faire adjectif, et même slogan préconisant implicitement un certain style de vie.

\subsection{Contraintes discursives et modes d'organisation textuelle}

Il n'est pas exclu, par ailleurs, que le constant renvoi au passé et à la tradition fasse aussi peser de fortes contraintes discursives sur les modes d'organisation textuelle de certains genres écrits. Il est vrai que les genres relevant de la correspondance officielle (lettre, message, mémorandum...) paraissent strictement normés. Mais si nous reprenons l'exemple de l'article doctrinal, dont la grande liberté de ton et d'organisation fait tout l'intérêt, on peut se demander également si ce genre ne subit pas également des contraintes discursives d'ordre culturel. Certes, les officiers écrivant ces articles pour des revues prestigieuses telles que Parameters, Proceedings, Joint Forces Quarterly, n'hésitent pas - ils y sont même encouragés - à remettre en cause les paradigmes officiels (dans la mesure toutefois où ils respectent un certain devoir de réserve et avancent des propositions constructives). En première approche, cependant, et sous réserve d'un dépouillement systématique d'un corpus d'articles doctrinaux, on peut avancer l'hypothèse que l'organisation textuelle de ces articles répond à un 
schéma contraint, qu'on pourrait rapprocher de la «jérémiade » décrite par $S$. Bercovitch au sujet des sermons puritains du XVII ${ }^{\mathrm{e}}$ siècle. Lorsqu'il s'agit d'avancer des propositions en vue d'améliorer l'armée d'aujourd'hui, l'auteur aura souvent recours à une structuration du texte en trois temps (dont le séquençage peut varier), décrite dans le tableau ci-dessous ; ici encore, le besoin de légitimation par le passé et la mémoire se manifeste avec force :

Figure 1. Le renvoi au passé dans les articles doctrinaux

\begin{tabular}{|l|l|l|}
\hline $\begin{array}{l}\text { exaltation d'un passé } \\
\text { mythique, où les valeurs de } \\
\text { référence du milieu militaire } \\
\text { triomphaient }\end{array}$ & $\begin{array}{l}\text { The dilemma is that we need to reshape our culture without } \\
\text { destroying traditions that have served us well in the past. }\end{array}$ \\
\hline $\begin{array}{l}\text { description } \\
\text { communauté ayant renoncé à } \\
\text { ces valeurs et subissant les } \\
\text { malheurs et tourments de } \\
\text { l'apostat }\end{array}$ & $\begin{array}{l}\text { Former Air Force chief of staff Gen Michael Dugan once } \\
\text { commented to me that the Air Force is producing a generation of } \\
\text { illerate truck drivers. He worried that officers who aspire to } \\
\text { senior leadership positions know a great deal about airplanes and } \\
\text { precious little about airpower. }\end{array}$ \\
\hline $\begin{array}{l}\text { exhortation à suivre le } \\
\text { chemin menant à la } \\
\text { réhabilitation des modèles } \\
\text { anciens }\end{array}$ & $\begin{array}{l}\text { Somehow, we must make it culturally acceptable and } \\
\text { professionally imperative to be air warriors well schooled in the } \\
\text { theory, doctrine, and history of aerial warfare. [...] Reshaping our } \\
\text { culture without destroying our traditions is the key to making the } \\
\text { next generation of Air Force leadership better than this } \\
\text { generation. (Drew) }\end{array}$ \\
\hline
\end{tabular}

41 Au-delà de l'effet de préconstruit dont nous avons tenté de montrer la prégnance, il est possible de penser que le renvoi à l'ancien et à la tradition se traduira par certains invariants, certaines régularités rhétoriques ou stylistiques dans de nombreux genres écrits propres aux militaires. Si cette hypothèse appelle une confirmation ultérieure par un travail de dépouillement plus systématique, il serait frappant de constater que, à l'instar du modèle «CARS » décrit par Swales (1990) au sujet de l'article scientifique, les textes doctrinaux des militaires américains manifesteraient par leur organisation l'étroit paramétrage induit par une culture partagée.

\section{Conclusion}

Non réductible à un simple conservatisme, l'attention portée à la mémoire et à la tradition au sein des milieux militaires américains permet, à travers des rituels vivaces et omniprésents, d'établir un bornage entre sphère civile et sphère militaire. Cristallisant des cultures locales fortement affirmées, tradition et mémoire sont également mobilisées pour délimiter des sous-ensembles au sein de ces milieux. Leur prégnance semble telle que, s'agissant de nombreuses productions langagières propres aux milieux militaires américains, tradition et mémoire semblent toujours en arrièreplan de la situation d'énonciation, au point de se transformer en préconstruit. Ce dernier peut être décrit comme un paramètre crucial de la situation d'énonciation, en tant qu'il matérialise par un faisceau d'attentes énonciatives l'inscription de chaque 
militaire américain dans un milieu professionnel historié de modèles hérités. Ainsi, dans de nombreux genres propres au monde militaire - articles doctrinaux, jeux langagiers, documents administratifs tels que le rapport de notation, le préconstruit paraît matérialiser l'appartenance de l'énonciateur à son milieu professionnel.

Aux mécanismes d'individuation induits par l'exaltation de la tradition et de la mémoire répondent donc des contraintes énonciatives qui, en adossant les productions langagières des militaires américains à la culture des forces armées (ou des sousensembles de celles-ci), en font un discours de spécialité. Dès lors, toute analyse de ce discours ne pourra se contenter de retracer ses caractéristiques de surface, mais devra tenter de décrire l'ensemble du dispositif énonciatif - y compris son versant culturel. Tout semble en effet indiquer que les milieux militaires américains répondent à la définition que donnait Balandier (1967) des sociétés gouvernées par un «traditionalisme formel»: l'institution, les cadres sociaux ou culturels, les représentations communes sont préservées, mais leur contenu est peu à peu modifié pour s'adapter aux circonstances du moment. Le lien social est ainsi perpétué, par un maintien des moyens, alors même que les buts et fonctions de la tradition partagée changent. Nous avons déjà tenté de montrer comment l'institution militaire recycle et récupère certains rites pour en faire des manifestations unitaires. Ce même mouvement peut être observé sur le plan énonciatif: les productions des militaires américains trouvent leur unité en tant que discours de spécialité dans ce rite que devient la stratégie de légitimation par l'ancien.

\section{BIBLIOGRAPHIE}

Balandier, G. 1967. Anthropologie politique. Paris : Presses universitaires de France.

Balandier, G. 1992. Le pouvoir sur scènes. Paris : Balland.

Bercovitch, S. 1980. The American Jeremiad. Madison, WI : University of Wisconsin Press.

Coonts, S. 1994. The Intruders. New York : Pocket Books.

Culver, C. (Capt.). 1992. « O Clubs: Tradition or Contradiction? ». Aerospace Power Journal 6/4, 46-59.

Drew, D. M. (Col.). 1997. «Educating Air Force officers ». Aerospace Power Journal 9/ 2, 37-44.

Dufoulon S. 1998. Les gars de la Marine, ethnographie d'un navire de guerre. Paris : Métailié.

Griffin, W. E. B. 1987. The New Breed (Brotherhood of War). New York : Jove Fiction.

Hall, S. C. 1987. « Shortchanging our young officers: Military traditions denied ». Aerospace Power Journal 1/2, 48-57.

Hall, T. (Col.). 2001. "“I'd rather be flying," The ethos of the Air Force officer corps ». Aerospace Power Journal 15/2.

Iversen, A (Capt.). 2001. « Professional military education for company grade officers: Targeting for "affect" ». Aerospace Power Journal 15/ 2, 58-64. 
Janowitz, M. 1960. The Professional Soldier: A social and political portrait. New York : Free Press.

Moskos, C. 1970. The American Enlisted Man. New York : Sage.

Naval Historical Center: Traditions of the Naval Service. Washington : United States Navy novembre $2002<$ http://www.history.navy.mil/nhc11.htm>.

Paveau, M. A. 1994. « Le langage des militaires ». Thèse de l'Université Paris 4.

Pêcheux, M. 1975. Les vérités de La Palice. Linguistique, sémantique, philosophie. Paris : Maspero.

Powell, C. L. 1995. My American Journey. New York : Ballantine.

Rivière, C. 1988. Les Liturgies politiques. Paris : Presses universitaires de France.

Swales, J. 1990. Genre Analysis. English in Academic and Research Settings. Cambridge : Cambridge University Press.

Thieblemont, A. (dir.). 1999. Culture et logiques militaires. Paris : Presses universitaires de France.

Vernon, A. et al. 1999. The Eyes of Orion, Five Tank Lieutenants in the Persian Gulf War. Kent : The Kent State University Press.

Waller, D. C. 2001. Big Red, The Three-Month Voyage of a Trident Nuclear Submarine. New York: Harpertorch.

\section{NOTES}

1. Vining. P., 2002, United States Naval Institute Proceedings 128/1, 78.

2. Duquette C., 2003, United States Naval Institute Proceedings129/1, 93.

3. Record, J., 2000, United States Naval Institute Proceedings126/10, 35.

4. Dunne, S., 2000, United States Naval Institute Proceedings126/12, 68.

\section{RÉSUMÉS}

$\mathrm{Au}$ sein de l'armée américaine, tradition et mémoire font l'objet d'un culte entretenu par l'institution militaire. Par la célébration du passé, les militaires américains construisent un patrimoine partagé, qui fournit le substrat d'une culture professionnelle vivace. Celle-ci se manifeste par des rites et des pratiques symboliques qui permettent à diverses " chapelles » au sein de l'institution militaire d'exprimer leur cohésion et leur esprit de corps. La prégnance de la mémoire et de la tradition se manifeste également au plan énonciatif, en contribuant à édifier un style communicatif spécifique. On note que la nécessaire adhésion à une culture professionnelle partagée suscite des "effets de préconstruit» qui affectent la situation d'énonciation et la sémantique des énoncés.

The American military worships its traditions and its past, which emerge as the cornerstone of military culture. The latter is based on rites and symbolic practices which allow different subcultures within the military to express a strong esprit de corps. The cult of the past also affects enunciative patterns, by imposing a specific communicative style on members of the military. 
The military's pervasive professional culture thus allows the "preconstructed" to surface, both in the enunciative situation and in utterance semantics.

INDEX

Mots-clés : culture professionnelle, préconstruit, situation d'énonciation

Keywords : enunciative situation, preconstructed, professional culture

\section{AUTEUR}

ANTHONY SABER

Anthony Saber a enseigné à l'École navale de 1996 à 2001. Agrégé d'anglais, ancien élève de l'École Normale Supérieure, cette expérience professionnelle l'a amené à s'intéresser aux milieux militaires américains et aux spécificités de l'anglais militaire. Il est actuellement affecté au département des langues de l'École Normale Supérieure de Cachan. saber.cachan@ens-cachan.fr 